\title{
Attitudes, training and smoking profile of European Respiratory Society members
}

\section{To the Editors:}

Patients regard healthcare professionals, particularly physicians, as role models for leading a healthy lifestyle. Healthcare professionals' own behaviour and attitudes concerning healthy lifestyles have an influence on the counselling of patients. A recent survey in the USA reported $\sim 6 \%$ tobacco use among healthcare professionals [1], while $41 \%$ of Chinese male medical doctors smoke [2]. Tobacco dependence treatment (TDT) efforts generally focus on physicians, but evidence suggests that physicians are inadequately trained to help smokers quit $[3,4]$. For a successful TDT programme, appropriately trained healthcare professionals are essential. The need and quality of training for TDT currently available for healthcare professionals across Europe is unknown.

The European Respiratory Society (ERS) is the leading professional organisation in its field in Europe (www.ersnet.org). ERS is broad-based, with $\sim 10,000$ members from 100 different countries working in the field of respiratory health. We undertook a telephone survey on smoking profiles of ERS members in Europe from November 2009 to January 2010, using a standardised questionnaire designed with the leadership of the Tobacco Control Committee of the ERS.

Three main outcome measures were examined among ERS members: 1) percentages of current and ever-smokers (smoked $>100$ cigarettes in their lifetime); 2) second-hand smoke (SHS) exposure; and 3) attitudes and training of the ERS members regarding TDT.

The estimated sample size was derived from 25 European countries across five different geographic regions proportionally representative of the ERS membership (Western Europe, the Mediterranean region, Scandinavia, Central and Eastern Europe, and the Baltic region), as shown in table 1. An estimated sample size of 722 subjects was based on an expected response rate of $50 \%$, with $95 \%$ confidence and a $5 \%$ margin of error. Respondents were recruited randomly using a list of ERS members provided by the ERS. Consent was sought from the ERS members and confidentiality was guaranteed by the research company (Melides Marketing Resources (MMR), Puygouzon, France; an independent body that undertook the survey) for each respondent of the survey. MMR adhere to the International Chamber of Commerce/ European Society for Opinion and Marketing Research International Code on Market and Social Research [5].

Both descriptive and logistic regression analyses were performed using SAS statistical software (version 9.1.2; SAS Institute, Cary, NC, USA) for covariate-adjusted smoking estimates with adjusted odds ratios (aORs) and 95\% confidence intervals.

Of the 722 ERS members surveyed, 32 (4.4\%, 95\% CI 2.9-5.9\%) were current smokers compared with $33.4 \%$ of the general population. $27.9 \%$ (95\% CI $24.7-31.3 \%$ ) of the members smoked $>100$ cigarettes in their lifetime (ever-smokers) and 23.5\% (95\%
CI 20.4-26.6\%) were ex-smokers. The findings showed regional differences (table 1).

$32.4 \%$ (95\% CI $28.9-35.8 \%)$ of ERS members sampled were exposed to SHS, but this also showed regional variations (table 1). The main areas of SHS exposure were public houses, nightclubs, public transport and at home. In multivariable analyses, members were at significantly higher risk of being a current or ever-smoker if they spent time with a smoker at home compared with not spending time with a smoker (aOR 8.74 (95\% CI 3.06-24.9) and aOR 2.41 (95\% CI 1.18-4.91), respectively). Scandinavian members were least likely to spend time with others who are smoking. Central and Eastern European healthcare professionals were proportionately more exposed to SHS at home. In general, members were 3.5-fold more likely to be current smokers if they spent time with a smoker compared with not spending time with a smoker following adjustment for age, sex, training status and regional variations (aOR 3.61, 95\% CI 1.65-7.91).

$77 \%$ (95\% CI 71-83\%) of ever-smokers started smoking before the age of $20 \mathrm{yrs}$. More than half of the former smokers smoked for $\geqslant 10$ yrs during their lifetime. The overall mean \pm SD age of smoking initiation was $18.9 \pm 4.7 \mathrm{yrs}$ (variance $22.43 \mathrm{yrs}$ ). More than $60 \%$ of current smokers had been smoking for $>20$ yrs. None of the current smokers were $>65$ yrs of age; the majority of current smokers were $\leqslant 35$ yrs of age.

After adjustment for age, training status, SHS exposure levels and regional variations, male ERS members were more likely to be current or ever-smokers than female ERS members (aOR 1.48 (95\% CI 0.64-3.44) and aOR 1.71 (95\% CI 1.15-2.49), respectively). Members in Eastern and Central Europe were 2.5-fold more likely to be ever-smokers compared with those in Western Europe (aOR 2.59, 95\% CI 1.53-4.39). Similarly, 71\% increased ever-smoking rates were observed among the

\begin{tabular}{|c|c|c|c|c|c|}
\hline TABLE 1 & $\begin{array}{l}\text { European } \\
\text { sampled, } \\
\text { second-ha } \\
\text { geographic }\end{array}$ & $\begin{array}{l}\text { espirator } \\
\text { urrent an } \\
\text { d smoke } \\
\text { distributi }\end{array}$ & $\begin{array}{l}\text { Society } \\
\text { ever-sm } \\
\text { (SHS) ex } \\
\text { n }\end{array}$ & $\begin{array}{l}\text { RS) r } \\
\text { kers, } \\
\text { sure }\end{array}$ & $\begin{array}{l}\text { embers } \\
\text { id } \\
y\end{array}$ \\
\hline \multirow[t]{2}{*}{ Region } & \multicolumn{2}{|c|}{ ERS members $n$} & \multicolumn{2}{|c|}{ Smokers \% } & \multirow{2}{*}{$\begin{array}{c}\text { SHS } \\
\text { exposure \% }\end{array}$} \\
\hline & Registered & Sampled & Current & Ever & \\
\hline Western & 2828 & 344 & 4.1 & 23.8 & 23.5 \\
\hline Mediterranean & 2100 & 182 & 3.9 & 32.9 & 44.5 \\
\hline Scandinavian & 498 & 62 & 0 & 22.6 & 8.1 \\
\hline $\begin{array}{l}\text { Central/ } \\
\text { Eastern }\end{array}$ & \multicolumn{4}{|c|}{ Eastern } & 53.7 \\
\hline Baltic & 190 & 26 & 3.9 & 11.5 & 30.8 \\
\hline Total & 6501 & 722 & 4.4 & 27.9 & 32.4 \\
\hline
\end{tabular}


members in the Mediterranean region compared with those in Western Europe (aOR 1.71, 95\% CI 1.12-2.62).

Regarding attitudes on and training for TDT, almost all respondents (97.8\%, 95\% CI 96.7-98.9\%) felt that it was their responsibility to advise patients about smoking cessation, but only $35.4 \%$ (95\% CI $31.9-38.9 \%$ ) said they had formal training. This percentage also varied by region, being highest in the Mediterranean region (54\% versus 30\% in West Europe). Several members said that they had performed their own research and self-training to become informed. Few had training, or taught on training courses at their hospital or university. Many who had no formal training said they would be interested in participating in a training course. After adjustment for age, sex, SHS exposure levels and regional variations, training status was not found to be a significant predictor of either being a current or an ever-smoker. However, after simultaneous adjustment, those members who had some sort of training were less likely to be a current or ever-smoker (aOR 0.73 (95\% 0.32-1.66) and aOR 0.96 (95\% CI 0.67-1.39), respectively).

Of the 28 respondents who smoked, 10 (35.7\%, 95\% CI $16.8-$ $54.6 \%$ members regarded themselves as role models against smoking. $44 \%$ of current smokers had tried to quit in the previous year and of those who tried to quit, one-third used some form of smoking cessation but none used behavioural support as a smoking cessation tool.

A low rate of $4.4 \%$ current smokers among the ERS members is reassuring but the distinct geographic variations reflect that there may be a need for targeted comprehensive tobacco control programmes across Europe. A third of the healthcare professionals were also exposed to SHS. The fact that members exposed to SHS are significantly more likely to be current smokers reinforces the need for comprehensive smoke-free policies across Europe. It is interesting to note that members exposed to SHS at home are also at significantly higher risk of being a current smoker. Such observations indicate that smoke-free policies should expand beyond enclosed spaces and encourage voluntary smoke-free home policies [6]. In the USA, public health law researchers recently recommended the use of federal regulatory or contractual mechanisms to ensure that public housing authorities implement no-smoking policies for the protection of children and others [7], following strong encouragement of such policies expressed in a July 2010 memorandum by the US Dept of Housing and Urban Development [8].

Furthermore, sex variations in smoking rates were also noted. Male ERS members were at significantly higher risk of being ever-smokers. Although a higher proportion of ERS members are males, this sex difference in smoking rates was based on multivariate analyses. In addition, smoking ERS members were young, started smoking before the age of 20 yrs and had been smoking for $>20$ yrs. It is also worrying that more than half of the current smokers did not want to quit smoking in the near future and of those who did want to quit, less than half of them use any of the existing proven smoking cessation approaches.

The survey also indicated the unmet need for TDT education in the ERS, consistent with the ERS Task Force on smoking cessation guidelines [9]. ERS members overwhelmingly wished to treat tobacco dependence but only a minority were trained adequately to do so. Although training status did not significantly predict current smoking status, those who had some form of training were less likely to smoke. It is also interesting to note that one-third of currently smoking members regarded themselves as role models against smoking, further highlighting the need for adequate training and awareness programmes.

This was a cross-sectional survey and smoking status was selfreported; thus, biases are possible, but similar studies across other major European societies, such as the European Cardiac Society, should be conducted to capture a more comprehensive smoking landscape of healthcare professionals in Europe. No distinctions between different categories of healthcare professionals were feasible within the ERS membership registration system but only $15 \%$ of the ERS members surveyed were nonmedical members.

Some worrying trends were noted in this baseline survey, such as the need for holistic, comprehensive smoke-free policies that might include voluntary smoke-free home policies [6]. However, from an ERS perspective, the immediate concern should focus on training our members in TDT [9]. In order to ensure that they are equipped to fulfil the role of leaders in the battle to reduce the toll of death and morbidity from smoking-related respiratory disease to which they aspire in their professional life [10], ERS healthcare professionals should be supported in their positions as role models for a healthy smoke-free lifestyle.

\section{Z. Kabir*, B. Ward" and L. Clancy* on behalf of the Tobacco Control Committee of the European Respiratory Society \\ *TobaccoFree Research Institute, Dublin, Ireland. "European Respiratory Society, Brussels, Belgium.}

Correspondence: L. Clancy, TobaccoFree Research Institute, The Digital Depot, Thomas Street, Dublin 8, Ireland. E-mail: lclancy@tri.ie

Support Statement: This study was funded by the European Respiratory Society.

Statement of Interest: None declared.

Acknowledgements: We would like to thank the ERS members for their cooperation and Melides Marketing Resources, especially J. Gould, for the telephone survey.

\section{REFERENCES}

1 Tong EK, Strouse R, Hall J, et al. National survey of US health professionals' smoking prevalence, cessation practices, and beliefs. Nicotine Tob Res 2010; 12: 724-733.

2 Jiang Y, Ong MK, Tong EK, et al. Chinese physicians and their smoking knowledge, attitudes, and practices. Am J Prev Med 2007; 33: $15-22$.

3 Thorndike AN, Regan S, Rigotti NA. The treatment of smoking by US physicians during ambulatory visits: 1994-2003. Am J Public Health 2007; 97: 1878-1883.

4 Ferketich AK, Khan Y, Wewers ME. Are physicians asking about tobacco use and assisting with cessation? Results from the 20012004 national ambulatory medical care survey (NAMCS). Prev Med 2006; 43: 472-476. 
5 International Chamber of Commerce, European Society for Opinion and Marketing Research. ICC/ESOMAR International Code On Market and Social Research www.esomar.org/index. php/codes-guidelines.html Date last updated: December 2007.

6 Heck JE, Stücker I, Allwright S, et al. Home and workplace smoking bans in Italy, Ireland, Sweden, France and the Czech Republic. Eur Respir J 2010; 35: 969-979.

7 Winickoff JP, Gottlieb M, Mello MM. Regulation of smoking in public housing. New Eng J Med 2010; 362: 2319-2325.

8 Dept of Housing and Urban Development, Office of Public and Indian Housing. Non-Smoking Policies in Public Housing
(Memorandum). www.hud.gov/offices/pih/publications/notices/ 09/pih2009-21.pdf Date last accessed: June 27, 2010. Date last updated: July 17, 2009.

9 Tønnesen P, Carrozzi L, Fagerström KO, et al. Smoking cessation in patients with respiratory diseases: a high priority, integral component of therapy. Eur Respir J 2007; 29: 390-417.

10 Sigsgaard T, Clancy L, Forastiere F, et al. 20 years of research and advocacy for a healthy and tobacco-free environment. Eur Respir $J$ 2010; 36: 1-3.

\section{Shorter treatment duration for selected patients with multidrug-resistant tuberculosis}

\section{To the Editors:}

Tuberculosis (TB) is still an infectious disease of public health importance today [1]. Of particular concern is the occurrence of multidrug-resistant (MDR)-TB with resistance to at least isoniazid and rifampicin, and extensively drug-resistant (XDR)-TB with additional resistance to any fluoroquinolone and at least one of the second-line injectable drugs, including kanamycin, amikacin and capreomycin. MDR- and XDR-TB need prolonged treatment duration, from 18 to 24 months after sputum culture conversion, as recommended by the World Health Organization (WHO) [2]. A prolonged duration of treatment may lead to poor adherence, higher cost and undue toxicity. In our previous studies, we found that the use of ofloxacin/levofloxacin-containing regimens had a favourable effect on MDR-TB outcome [3, 4]. Our studies also suggested that shorter treatment duration (mean duration 14 months) might be feasible in selected MDR-TB patients. Based on this finding, the treatment duration of selected MDR-TB patients under programmatic management in Hong Kong was changed from the rigid enforcement of $\geqslant 18$ months after sputum conversion to an individualised duration tailored by the physician's judgement. The determinants favouring longer treatment included extensive drug resistance, delayed sputum culture conversion, greater extent of radiographic disease (including cavities), presence of diabetes or silicosis, and extrapulmonary disease. Out of these factors, the extent of drug resistance was the most important. Ofloxacin-resistant MDRTB was arbitrarily given a prolonged treatment based on our previous studies $[3,4]$. Despite a relatively high TB incidence (76 cases per 100,000 population in 2009), both TB and MDR$\mathrm{TB}$ rates are actually decreasing under the local TB control programme [5]. Therefore, we conducted a retrospective evaluation of the long-term outcomes of our MDR-TB patients, focusing on the factors affecting the efficacy and relapse rate of a shorter treatment duration.

All consecutive MDR-TB cases under the programmatic management of the Hong Kong Tuberculosis and Chest Service from 1997 to 2006 were included in this retrospective cohort. Case categories and clinical outcomes at 2 yrs were classified according to the criteria proposed by LASERSON et al. [6]. The final clinical status of successful patients (cured or having completed treatment) and the survival status of the entire cohort were ascertained until December 31, 2009. Patients lost to follow-up were tracked through the territorywide TB notification registry and death registry, which captured TB cases and deaths, respectively, all over Hong Kong. For analysis of survival and relapse, incidence densities were compared between different categories in univariate analysis, followed by Cox proportional hazard analysis in multivariate analysis. Statistical analyses were performed with EPI Info version 6.04 (Centers for Diseases Control and Prevention, Atlanta, GA, USA) and SPSS version 16 (IBM, Quarry Bay, Hong Kong). A two-tailed p-value $<0.05$ was taken as statistically significant.

The study was approved by the Ethics Committee of the Department of Health, Hong Kong. All patients were informed at the time of care delivery that the clinical data collected would be used for statistical analysis and research purposes.

There were 270 MDR-TB patients (including 27 XDR-TB patients) in the cohort. They included $245(90.7 \%)$ patients with pulmonary TB only, six (2.2\%) patients with extrapulmonary TB only, and 19 (7.1\%) patients with both pulmonary and extrapulmonary involvement. Voluntary HIV testing was offered to all patients, with 45 declining the test. Three $(1.2 \%)$ out of 235 patients tested were HIV-positive and they were all non-XDR, MDR-TB patients.

Of all included subjects, $187(69.3 \%)$ patients were cured or completed treatment, $26(9.6 \%)$ died during treatment, $45(16.7 \%)$ defaulted on treatment (with median treatment duration 5 months, range $0.5-18$ months), nine (3.3\%) were transferred out and three $(1.1 \%)$ had treatment failure. All failure patients had XDR-TB. Lung resections (eight lobectomies, one lobectomy plus segmentectomy, and one pneumonectomy) were performed in a total of 10 patients (three XDR-TB and seven non-XDR, MDR-TB).

Among patients successfully treated, significantly longer duration of treatment was associated with XDR-TB compared with other MDR-TB patients (median duration 21 versus 15 months; $\mathrm{p}<0.01$ ). $55.1 \%$ (97 out of 176 ) of the successfully treated non-XDR, MDR-TB patients were treated for $\leqslant 15$ months (median 13.5 months, range 11-15 months). 